\title{
Antifungal Activity of Osthole on Microsporum canis through Interfering with Biosynthesis of Fungal Cell Wall
}

\author{
C. Y. LIU, R. YANG, P. JIANG, T. T. SUN, T. ZHANG ${ }^{1}$ AND C. Y. HAN*
}

College of Animal Science and Technology, Anhui Agricultural University, 130 West Changjiang Road, Shushan District, Anhui 230036, ${ }^{1}$ College of Animal Science and Technology, Beijing University of Agriculture, 7 Beinong Road, Changping District, Beijing 102206, P. R. China

\section{Liu et al.: Antifungal Effect of Osthole on Microsporum canis}

\begin{abstract}
Osthole extracted from Cnidii Fructus, a herb used in traditional Chinese medicine, was reported to possess multiple activities such as antibacterial, antiinflammatory, antitumor and insecticidal. However, its antifungal activity against Microsproum canis has not been investigated yet. The present study aimed to investigate the antifungal effect of osthole against Microsproum canis. The minimum inhibitory concentration and a growth inhibitory curve of osthole on Microsporum canis were according to the program of M38-A2 of Clinical and Laboratory Standards Institute. High-performance liquid chromatography analysis, aniline blue test and spectrophotometry were used to determine contents of ergosterol, 1,3- $\beta$-D-glucan and chitin from Microsporum canis, respectively. The results indicated that minimum inhibitory concentration of osthole against Microsporum canis was $1.95 \mu \mathrm{g} / \mathrm{ml}$. High-performance liquid chromatography analysis demonstrated that there was no significant change in the content of ergosterol between the osthole and control group. Compared with the control group, the content of 1,3- $\beta$-D-glucan and chitin in the cell wall of Microsporum canis was decreased. Taken together, this research showed that osthole could notably inhibit the growth of Microsporum canis and the antifungal activity was related to inhibiting the biosynthesis of 1,3- $\beta$-D-glucan and chitin in the cell wall of Microsporum canis, making osthole a potential future antifungal agent.
\end{abstract}

Key words: Osthole, Microsporum canis, ergosterol, cell wall, 1,3- $\beta$-D-glucan, chitin

Microsporum canis is one of the common and epidemic pathogenic fungi worldwide that can invade keratinized structures and infect hair, skin and nails of humans and animals. Furthermore, $M$. canis is zoonotic in nature and highly contagious to Feline, Canis and Homo sapiens ${ }^{[1,2]}$. The animals could be infected by $M$. canis during each season, notably, it has the highest incidence rate in the hot and damp seasons ${ }^{[3]}$. The pathogen causing $60 \%$ of fungal skin diseases of canis and $98 \%$ dermatophytes diseases of feline was found to be $M$. canis $^{[4]}$. Once the animals infected by $M$. canis, the disease is hard to cure since it relapses easily and may generate cross-infection with other pathogens, which is characterized by increasing furfur, forming scab, exuding body fluid, pruritus and so on ${ }^{[5,6]}$.

Osthole, 7-methoxy-8-(3-methyl-2-butenyl) coumarin (fig. 1), is a coumarin derivative extracted from Cnidii Fructus, a traditional Chinese medical herb ${ }^{[7]}$. Previous studies have showed that osthole could exert antibacterial, antiinflammatory, antitumor and insecticidal activities, relieve heart disorder, dilate blood

*Address for correspondence E-mail: luckyhcy@163.com vessels and lower blood pressure ${ }^{[8-12]}$. A recent report showed that osthole could prevent the reproduction of Alternaria and Cryptococcus neoformans ${ }^{[13]}$. However, there is no investigation focusing on the antifungal activity and the possible targets of antifungal properties of osthole. Therefore, this preliminary assessment to investigate the inhibitory effect of osthole on $M$. canis was taken up.

\section{MATERIALS AND METHODS}

Osthole ( $>98 \%$ HPLC purity) was purchased from Nanning Innovative Pharmaceutical Technology Co., Ltd. (Guangxi, China). Caspofungin was obtained from New Pioneer Pharmaceutical Company (Shanghai, China). Terbinafine was purchased from Dalian Meilun

This is an open access article distributed under the terms of the Creative Commons Attribution-NonCommercial-ShareAlike 3.0 License, which allows others to remix, tweak, and build upon the work non-commercially, as long as the author is credited and the new creations are licensed under the identical terms

Accepted 06 August 2018

Revised 03 February 2018

Received 30 May 2017

Indian J Pharm Sci 2018;80(5):852-857 


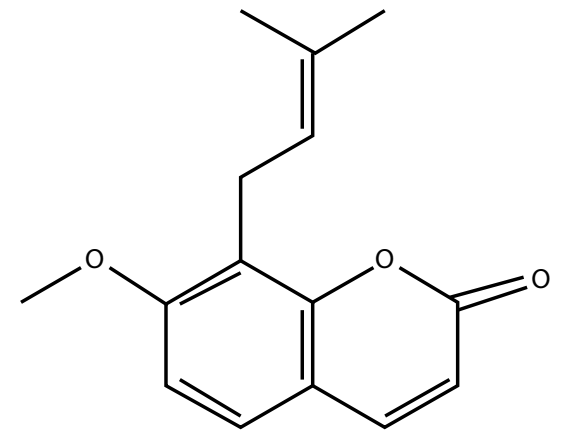

Fig. 1: Chemical structure of osthole

Bio-Technology Co., Ltd. (Dalian, China). Ergosterol, aniline blue and dimethyl sulfoxide (DMSO) were purchased from Sigma-Aldrich Chemicals (St. Louis, MO, USA). Sabouraud dextrose agar (SDA) was purchased from Qingdao Hope BioTechnology (Shandong, China). RPMI-1640 liquid medium was purchased from Yuan Ye BioTechnology Co., Ltd. (Shanghai, China). Other reagents were obtained from Sinopharm Chemical Reagent Co. Ltd., Shanghai Shi, China.

\section{Fungi culture:}

Microsporum canis (YUMCC Ym3099) was provided by the laboratory of fungi of Department of Clinical Veterinary Medicine at China Agricultural University in Beijing, China. M. canis was grown on SDA and incubated at $28^{\circ}$ for $7 \mathrm{~d}$, then picked single colony and cultured in Sabouraud dextrose broth. The concentration of $M$. canis was adjusted to $5 \times 10^{5}$ colony forming units (CFU)/ml using RPMI-1640 medium, and this suspension was used as inocula for the following inhibition assays.

\section{Determination of minimum inhibitory concentration (MIC):}

The MIC was determined with broth microdilution methods based on the reference document M38-A2 for yeast and filamentous fungi formulated by the Clinical and Laboratory Standard Institute (CLSI) ${ }^{[14]}$. Osthole, terbinafine and caspofungin were dissolved in DMSO and further diluted to final concentrations with RPMI1640 medium. The final concentrations of osthole, terbinafine and caspofungin were 15.6, 0.8 and $0.16 \mu \mathrm{g} / \mathrm{ml}$, respectively. The final DMSO concentration was $\leq 0.1 \%(\mathrm{v} / \mathrm{v})$. One hundred microliters of RPMI1640 broth was added into each well of 96-well microtiter plates (Corning, NY, USA). Then either 100 $\mu 1$ osthole solution, terbinafine solution or caspofungin solution were added to the first well, and the multiple proportion dilution was conducted to obtain different final drug concentrations. The above wells were supplemented with $100 \mu 1$ spore suspension $\left(5 \times 10^{5} \mathrm{CFU} /\right.$ $\mathrm{ml}$ ) and for controls additional wells were only added to $200 \mu 1$ RPMI-1640 medium, respectively. The plates were incubated for $7 \mathrm{~d}$ at $28^{\circ}$ and performed in triplicate at each concentration. The absorbance at $620 \mathrm{~nm}$ was measured with a microplate reader (Bio-Rad, Foster, California, USA).

Inhibition curves of osthole, terbinafine and caspofungin on M. canis:

M. canis $\left(5 \times 10^{5} \mathrm{CFU} / \mathrm{ml}\right)$ was treated with osthole, terbinafine and caspofungin at MIC concentrations during the logarithmic phase of its growth. After shaking for $0,1,2,4,8,12,24,36,48$ and $72 \mathrm{~h}$ at $28^{\circ}$, the inhibitory effect of the compounds on $M$. canis was measured with the aid of a haemocytometer and growth inhibition curves were plotted.

High-performance liquid chromatography (HPLC) analysis:

M. canis was treated during logarithmic phase of its growth with osthole, terbinafine and caspofungin at MIC concentration for $24 \mathrm{~h}$, respectively. After $7 \mathrm{~d}$ of incubation, $M$. canis hyphae was harvested by centrifugation at $1300 \mathrm{rpm}$ for $15 \mathrm{~min}$ and washed twice with phosphate buffered saline at room temperature. The hyphae was collected, and resuspended in $15 \mathrm{ml}$ saponifier and then incubated at $80^{\circ}$ for $90 \mathrm{~min}$. Then, $15 \mathrm{ml}$ petroleum ether was added and incubated for $60 \mathrm{~min}$. After being dried, the non-saponifiable extract was collected dissolved with methanol, and filtered with a Millipore filter of $0.22 \mu \mathrm{m}$. The aliquot was used for HPLC detection ${ }^{[15]}$.

The condition of chromatographic detection was $284 \mathrm{~nm}$ at $1 \mathrm{ml} / \mathrm{min}$ flow rate with $100 \%$ methanol as mobile phase and column temperature at $35^{\circ[15]}$. Standard solution with different concentrations of ergosterol was prepared, and the sample was determined under the above chromatographic conditions. The standard curve of ergosterol was drawn by setting the concentration as abscissa and the peak area as the vertical. The resultant precision, stability and recovery of the HPLC methods were evaluated.

\section{Measurement of 1,3- $\beta-D$-glucan:}

The content of 1,3- $\beta$-D-glucan was determined using aniline blue assay ${ }^{[16]}$. Briefly, $50 \mu \mathrm{l}$ suspensions of $M$. canis was added into $10 \mathrm{ml}$ RPMI-1640 medium containing osthole or caspofungin at MIC 
concentration, respectively. After incubation at $28^{\circ}$ for $7 \mathrm{~d}$, the $M$. canis was collected by centrifugation at $1300 \mathrm{rpm}$ for $3 \mathrm{~min}$, then washed twice with $0.1 \mathrm{M}$ $\mathrm{NaOH}$ solution and freeze-dried for $16 \mathrm{~h}$. A total of $5 \mathrm{mg}$ M. canis was added into $250 \mu 11 \mathrm{M} \mathrm{NaOH}$ solution. The mixture was blended for $30 \mathrm{~s}$ and incubated for $30 \mathrm{~min}$ at $52^{\circ}$. A volume of $50 \mu 1$ the above mixture and $185 \mu \mathrm{l}$ aniline blue $(0.067 \%$ aniline blue, $0.35 \mathrm{M}$ $\mathrm{HCl}, 0.98 \mathrm{M}$ glycine- $\mathrm{NaOH}, \mathrm{pH} 9.5)$ were added into 96-well plates with triplicate and then incubated for $30 \mathrm{~min}$ at $52^{\circ}$. Absorbance was measured in a microplate reader (Bio-Rad, Foster, California, USA) at excitation/emission $410 / 458 \mathrm{~nm}$.

\section{Measurement of chitin:}

The content of chitin was determined as previously described $^{[14]}$. A volume of $50 \mu 1$ suspensions of $M$. canis was added into $10 \mathrm{ml}$ RPMI-1640 medium containing osthole or caspofungin at MIC concentration, respectively. After incubation at $28^{\circ}$ for $7 \mathrm{~d}$, the M. canis was collected by centrifugation at $1300 \mathrm{rpm}$ for $3 \mathrm{~min}$, then washed twice with $0.1 \mathrm{M} \mathrm{NaOH}$ solution and freeze-dried for $16 \mathrm{~h}$. Briefly, $5 \mathrm{mg}$ M. canis was resuspended in $3 \mathrm{ml} \mathrm{KOH}$ saturated solution, incubated for $1 \mathrm{~h}$ at $130^{\circ}$, added $8 \mathrm{ml} 75 \%$ ice-cold ethanol for $15 \mathrm{~min}$ in ice bath, and then mixed with a volume of $300 \mu \mathrm{l}$ diatomite 545 suspension (13.3\%), and centrifuged at $2000 \mathrm{rpm}$ for $5 \mathrm{~min}$ at $2^{\circ}$. The precipitate was washed once with $10 \mathrm{ml}$ ice-cold ethanol (40\%), and then washed twice with $10 \mathrm{ml}$ ice-cold water. The precipitate was resuspended in $500 \mu 1$ sterile distilled water, $500 \mu \mathrm{l} \mathrm{NaNO}$ (5\%) and $500 \mu 1 \mathrm{KHSO}_{4}(5 \%)$, and then centrifuged at $2000 \mathrm{rpm}$ for $2 \mathrm{~min}$ at $2^{\circ}$. After centrifugation, $150 \mu 1$ supernatant was transferred to sterile tubes and then $450 \mu \mathrm{l}$ sterile distilled water, $200 \mu \mathrm{l}$ aqueous ammonia (12.5\%) and $200 \mu 15 \mathrm{mg} / \mathrm{ml}$ 3-methylbenzothiazolinone-2-hydrazone were added and incubated for $3 \mathrm{~min}$ at $130^{\circ}$. The mixture was procedurally cooled down to room temperature, and $200 \mu 10.83 \% \mathrm{FeCl}_{3}$ was added, followed by incubation for $25 \mathrm{~min}$ at room temperature. Every sample was detected at $650 \mathrm{~nm}$ using spectrophotometer.

\section{Statistical analysis:}

Results from a representative of three independent experiments were expressed as means \pm standard deviation (SD), and analysed using the one-way analysis of variance (ANOVA) followed by least significant difference test for multiple comparisons, and post-hoc test using SPSS 21.0 (SPSS, Inc., Chicago, IL, USA).

\section{RESULTS AND DISCUSSION}

At present, synthetic drugs, including triazoles, polyenes and others, are being widely used for treating $M$. canis infection. With the wide application of these drugs as antifungal agents multidrug resistant fungi emerged on the clinical front ${ }^{[16]}$. Besides, the synthetic drugs also possessed certain deficiencies such as a poor curative effect on antifungal therapy ${ }^{[17]}$. Sensitivity to triazoles became reduced due to the deficiency of $A m B$ binding sites, which could inhibit ergosterol biosynthesis ${ }^{[18]}$. Polyenes could inhibit the growth of fungi by interacting with ergosterol in cell membrane, but are ineffective on fungus without ergosterol in cell membrane ${ }^{[19]}$. Although the drugs designed for the cell wall of fungi such as caspofungin, micafungin and anidulafungin, have acquired corresponding progress, the drug species has been limited ${ }^{[17]}$. Therefore, in recent years natural product and active ingredient contained from those have attracted more and more attention, which could provide a new choice for the treatment of fungal diseases.

In this study, the results indicated that that MIC of osthole, terbinafine and caspofungin against $M$. canis were $1.95,0.10$ and $0.02 \mu \mathrm{g} / \mathrm{ml}$, respectively. The number of viable fungi was not changed after treated with MIC of osthole, terbinafine and caspofungin for $1,2,4,8$, and $12 \mathrm{~h}$. After being stimulated with osthole for $24 \mathrm{~h}$, the number of viable fungi was significantly inhibited, and the effect of viable $M$. canis inhibition induced by osthole for $36 \mathrm{~h}$ was more serious. An obvious decrease in the number of viable fungi occurred after treatment with terbinafine and caspofungin for $24 \mathrm{~h}$ (fig. 2). The number of viable fungi significantly decreased with passage of time of exposure to osthole, terbinafine and caspofungin and declined to zero rapidly in the three groups, which suggested that osthole could absolutely inhibit the growth of M. canis within $36 \mathrm{~h}$ and exert the antifungal effect on $M$. canis. The results indicated that $1.95 \mu \mathrm{g} / \mathrm{ml}$ osthole and $0.02 \mu \mathrm{g} / \mathrm{ml}$ caspofungin could inhibit the growth of $M$. canis absolutely within $36 \mathrm{~h}$, while the time of terbinafine inhibit the growth of $M$. canis absolutely was significantly greater than that of osthole. It was suggested that osthole has a good prospect in the treatment of infectious disease of M. canis.

Ergosterol, the main steroidal substance in the fungal cell membrane, can maintain integrity, viability and normal growth of fungi. In addition, ergosterol can 


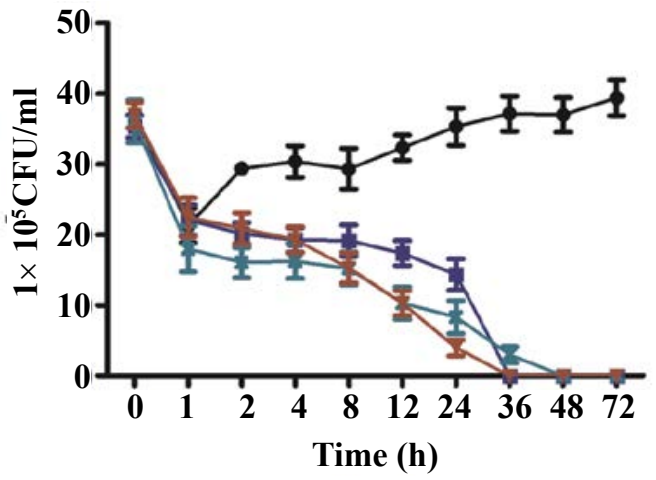

Fig. 2: Inhibitory curves of osthole, terbinafine and caspofungin on Microsporum canis

The logarithmic phase of $M$. canis $\left(5 \times 10^{5} \mathrm{CFU} / \mathrm{ml}\right)$ was exposed to MIC concentrations of osthole, terbinafine and caspofungin for $0,1,2,4,8,12,24,36,48$ and $72 \mathrm{~h}$, respectively. Data represent means \pm SD of 3 independent determinations and differences between mean values were assessed by oneway ANOVA. - - Control group; - - osthol group; $-\times-$ terbinafine group; $-\nabla-$ caspofungin group

adjust cell membrane fluidity by combining with phospholipid ${ }^{[17,19]}$. The fungal cell might suffer fracture due to the lack of ergosterol ${ }^{[19]}$. Some agents exhibited antifungal activity by inhibiting biosynthesis of ergosterol or interacting with ergosterol, and destroying the cell membrane structure of fungi ${ }^{[17,20]}$. Terbinafine, a polyene antifungal drug, was set as a standard inhibitor of ergosterol synthesis. The content of ergosterol was detected by HPLC after the interaction of osthole with M. canis. The standard curve equation of ergosterol obtained was $\mathrm{y}=1.7389 \mathrm{x}-2.5539(\mathrm{r}=0.999)$ under the liquid chromatograph condition. HPLC analysis results demonstrated that the content of ergosterol in osthole group and control group was significantly higher than that in terbinafine group, while no significant change was found in ergosterol content between osthole group and control group (figs. 3 and 4). The results demonstrated that osthole did not inhibit ergosterol biosynthesis in the cell membrane, which suggested that the antifungal activity of osthole could be related to the synthesis of cell wall.

The fungi is endowed with a cell wall to maintain its cell shape and transmit the signals. The loss of cell wall structure or function can lead to the loss of cellular homeostasis, which ultimately leads to cell death. It was reported that the destruction of fungal cell wall might cause dissolution of fungal cells, which eventually cause fungal death ${ }^{[17,21]}$. Therefore, the fungal cell wall is the ideal targets of antifungal agents $^{[22]}$. The main components of the fungal cell walls are 1,3- $\beta$-D-glucan and chitin, which do not exist in mammalian cells. Some studies have shown that the transcription levels and activities of chitinase and chitin synthase were significantly increased during the transformation of yeast to hyphal form of Candida albicans $^{[23,24]}$. After cloning the chitinase gene and then performing site directed mutagenesis, the chitinase has obvious effect on both morphology and growth rate of mycelial and spore ${ }^{[25,26]}$. Moreover, chitinase plays an important physiological role in the building process of fungal cell wall, and its major function is to assist in synthesis of chitin synthase, and process and modify for synthesized chitin to achieve perfect space configuration of cell wall ${ }^{[27,28]}$. Thus, deficiency or insufficiency of chitinase will affect the normal growth of mycelium, and then influence the virulence of pathogenic fungi. 1,3- $\beta$-D-glucan has effect on maintaining the normal shape of the fungal cell, when the fungal invade the deep part of body, and then it is processed by macrophage, the lots of $1,3-\beta-\mathrm{D}-$ glucan will be released; thus, $1,3-\beta-D$-glucan could be considered as a target molecule of fungal infection in clinical examination ${ }^{[14]}$. Besides, it is also reported that 1,3- $\beta$-D-glucan, a fungal surface antigen, can cause a variety of immune responses in animal body ${ }^{[28,29]}$. In the past several decades, there appeared antifungal agent which can destroy cell walls by inhibiting the biosynthesis of chitin and 1,3- $\beta$-D-glucan, such as echinocandins and caspofungins ${ }^{[28]}$. To investigate the effects of osthole on fungal cell wall synthesis, we carried out specific test to detect the content of fungal $1,3-\beta-\mathrm{D}$ glucan and chitin. The content of $1,3-\beta-\mathrm{D}-$ glucan in both osthole group and caspofungin group was significantly lower than that in control group,

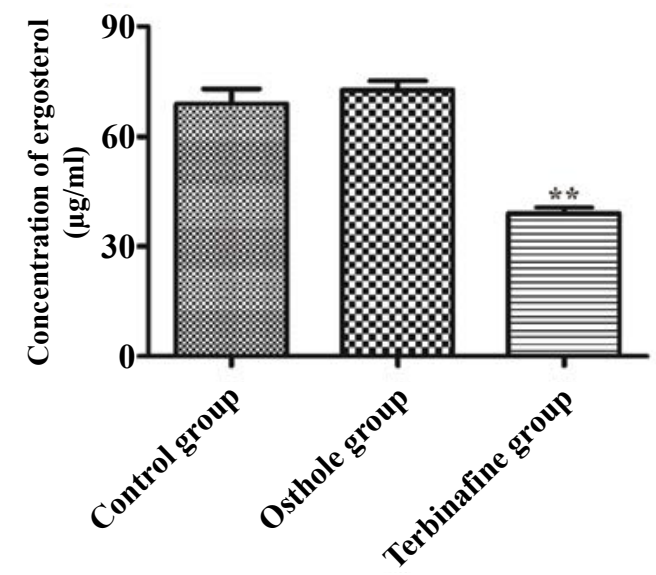

Fig. 3: Effect of osthole on ergosterol in Microsporum canis Effect of osthole on ergosterol content of $M$. canis was measured using HPLC assay. The logarithmic phase of $M$. canis $\left(5 \times 10^{5}\right.$ $\mathrm{CFU} / \mathrm{ml}$ ) was treated with MIC concentration of osthole and terbinafine for $24 \mathrm{~h}$, respectively. Data represent means \pm SD of three separate experiments and differences between mean values were assessed by one-way ANOVA. ${ }^{* *} \mathbf{P}<0.01$ indicates the significant difference compared with control group 
while no significant change was found in the 1,3- $\beta$-Dglucan content between osthole group and caspofungin group (fig. 5A). The data demonstrated that the content of chitin in both osthole group and caspofungin group was significantly decreased compared with the control group, while there was no significant difference in the content of chitin between osthole group and caspofungin group (fig. 5B). The results of this study indicated that the osthole has apparent inhibitory effect
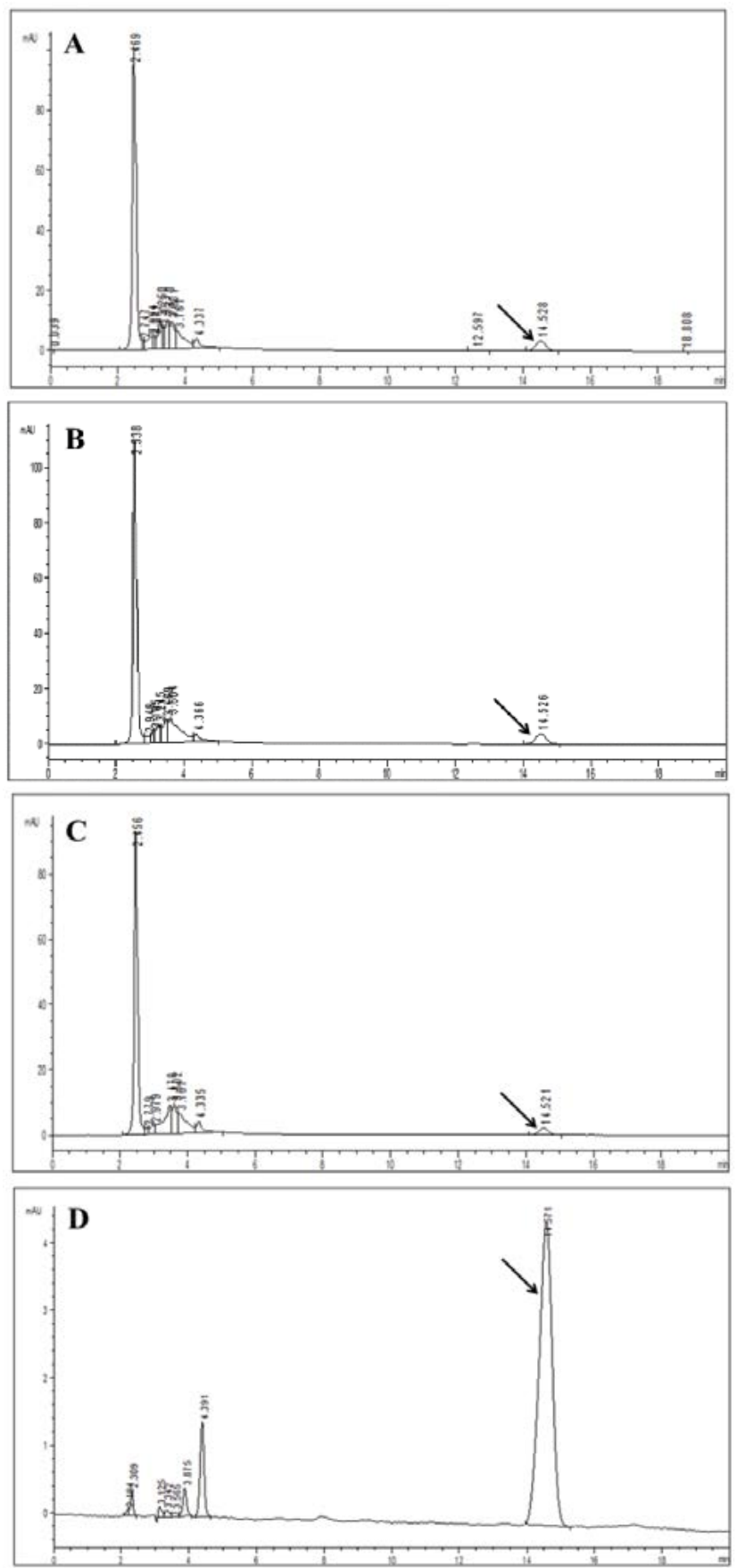

Fig. 4: HPLC chromatogram of ergosterol in Microsporum canis

A: Control group; B: osthole group; C: terbinafine group; D: ergosterol standard material.
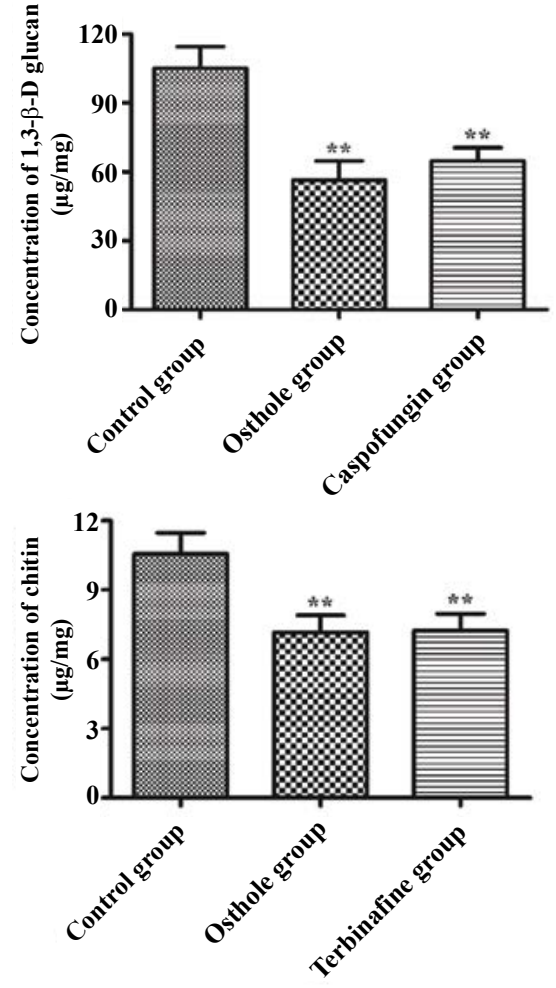

Fig. 5: Effect of osthole on 1, 3- $\beta$-D-glucan (A) and chitin (B) in Microsporum canis

Data represent means \pm SD of three separate experiments and differences between mean values were assessed by one-way ANOVA. ${ }^{* *} \mathbf{P}<0.01$ indicates the significant difference compared with control group

both on chitin and 1,3- $\beta$-D-glucan biosynthesis. Thus, osthole has stronger inhibition on inherent components of the M. canis cell wall.

In summary, our investigation demonstrated that osthole exerted an antifungal effect on $M$. canis by decreasing the content of $1,3-\beta-\mathrm{D}$ glucan and chitin, thereby interfering with the biosynthesis of fungal cell wall, and these results make a strong case that osthole could be a potential antifungal in place of the existing drugs.

\section{Acknowledgements:}

This work was financially supported by the National Natural Science Foundation of China (Grant No. 31772786) and the Open Project Program of Beijing Key Laboratory of Traditional Chinese Veterinary Medicine at Beijing University of Agriculture (TCVM 201701). We extremely thank Jingang $\mathrm{Gu}$ associate researcher for his kind help, and we also thank the Key Laboratory of Microbial Resource Collection and Preservation for providing the conditions for the investigation. 


\section{Conflict of interest:}

The author declares that there is no conflict of interest regarding the publication of this paper.

\section{REFERENCES}

1. Mancianti F, Nardoni S, Corazza M, D'Achille P, Ponticelli C. Environmental detection of Microsporum canis arthrospores in the households of infected cats and dogs. J Feline Med Surg 2003;5:323-8.

2. Hermoso de Mendoza M, Hermoso de Mendoza J, Alonso JM, Rey JM, Sanchez S, Martin R, et al. A zoonotic ringworm outbreak caused by a dysgonic strain of Microsporum canis from stray cats. Rev Iberoam Micol 2010;27:62-5.

3. Yin B, Xiao Y, Ran Y, Kang D, Dai Y, Lama J. Microsporum canis infection in three familial cases with Tinea capitis and Tinea corporis. Mycopathologia 2013;176:259-65.

4. Chah KF, Majiagbe KA, Kazeem HM, Ezeanyika O, Agbo IC. Dermatophytes from skin lesions of domestic animals in Nsukka, Enugu State, Nigeria. Vet Dermatol 2012;23:522e104.

5. Pasquetti M, Peano A, Soglia D, Min AR, Pankewitz F, Ohst T, et al. Development and validation of a microsatellite markerbased method for tracing infections by Microsporum canis. $\mathrm{J}$ Dermatol Sci 2013;2:123-9.

6. Cafarchia C, Figueredo LA, Coccioli C, Camarda A, Otranto D. Enzymatic activity of Microsporum canis and Trichophyton mentagrophytes from breeding rabbits with and without skin lesions. Mycoses 2012;1:45-9.

7. Chen Z, Mao X, Liu A, Gao X, Chen X, Ye M, et al. Osthole, a natural coumarin improves cognitive impairments and BBB dysfunction after transient global brain ischemia in C57 BL/6J mice: involvement of Nrf2 pathway. Neurochem Res 2015;40:186-94.

8. Yu C, Li P, Qi D, Wang L, Qu HL, Zhang YL, et al. Osthole protects sepsis-induced acute kidney injury via down-

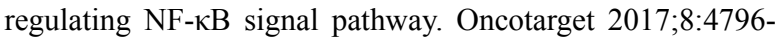
813.

9. Hao Y, Liu Y. Osthole alleviates bleomycin-induced pulmonary fibrosis via modulating angiotensin-converting enzyme 2/angiotensin-(1-7) axis and decreasing inflammation responses in rats. Biol Pharm Bull 2016;39:457-65.

10. Li Z, Ji H, Song X, Hu J, Han N, Chen N. Osthole attenuates the development of carrageenan-induced lung inflammation in rats. Int Immunopharmacol 2014;20(1):33-6.

11. Jiang G, Liu J, Ren B, Tang Y, Qwusu L, Li M, et al. Antitumor effects of osthole on ovarian cancer cells in vitro. J Ethnopharmacol 2016;193:368-76.

12. Liu M, Liu Y, Hua X, Wu C, Zhou S, Wang B, et al. Synthesis of osthole derivatives with grignard reagents and their larvicidal activities on mosquitoes. Chinese J Chem 2015;33:1353-58.

13. Xin XL, Dong PP, Wang G, Xi RG, Liu D, Wu ZM, et al. Biotransformation of osthole by alternaria longipes. J Asian Nat Prod Res 2013;15(7):717-22.

14. Fortwendel JR, Juvvadi PR, Pinchai N, Perfect BZ, Alspaugh JA, Perfect JR, et al. Differential effects of inhibiting chitin and $1,3-\beta$-D-glucan synthesis in ras and calcineurin mutants of Aspergillus fumigatus. Antimicrob Agents Chemother 2009;53(2):476-82.

15. Lopes G, Pinto E, Andrade PB, Valentão P. Antifungal activity of phlorotannins against dermatophytes and yeasts: approaches to the mechanism of action and influence on Candida albicans virulence factor. PLoS One 2013;8:e72203.

16. Sanglard D. Emerging treats in antifungal-resistant fungal pathogens. Front Med 2016;3:11.

17. Ostrosky-Zeichner L, Casadevall A, Galgiani JN, Odds FC, Rex JH. An sight into the antifungal pipeline: selected new molecules and beyond. Nat Rev Drug Discov 2010;9(9):719-27.

18. Sui G, Zhang W, Zhou K, Li Y, Zhang B, Xu D, et al. Trialkylamine derivatives containing a triazole moiety as promising ergosterol biosynthesis inhibitor: design, synthesis, and antifungal activity. Chem Pharm Bull 2017;65(1):82-9.

19. Récamier KS, Hernández-Gómez A, González-Damián J, Ortega-Blake I. Effect of membrane structure on the action of polyenes: I. Nystatin action in cholesterol- and ergosterolcontaining membranes. J Membr Biol 2010;237(1):31-40.

20. Ene IV, Adya AK, Wehmeier S, Brand AC, MacCallum DM, Gow NA, et al. Host carbon sources modulate cell wall architecture, drug resistance and virulence in a fungal pathogen. Cell Microbiol 2012;14(9):1319-35.

21. Levitz SM, Huang H, Ostroff GR, Specht CA. Exploiting fungal cell wall components in vaccines. Semin Immunopathol 2015;37(2):199-207.

22. Liu J, Balasubramanian MK. 1,3-beta-Glucan synthase: a useful target for antifungal drugs. Curr Drug Targets Infect Disord 2001;1:159-69.

23. Kelly MT, MacCallum DM, Clancy SD, Odds FC, Brown AJ, Butler G. The Candida albicans CaACE2 gene affects morphogenesis, adherence and virulence. Mol Microbiol 2004;53(3):969-83.

24. Selvaggini S, Munro CA, Paschoud S, Sanglard D, Gow NA. Independent regulation of chitin synthase and chitinase activity in Candida albicans and Saccharomyces cerevisiae. Microbiology 2004;150:921-8.

25. Ohnuma $T$, Taira $T$, Yamagami $T$, Aso $Y$, Ishiguro $M$. Molecular cloning, functional expression, and mutagenesis of cDNA encoding class I chitinase from rye (Secale cereale) seeds. Biosci Biotechnol Biochem 2004;68(2):324-32.

26. Tjoelker LW, Gosting L, Frey S, Hunter CL, Trong HL, Steiner B, et al. Structural and functional definition of the human chitinase chitin-binding domain. J Biol Chem 2000;275:514-20.

27. Chaudhary PM, Tupe SG, Deshpande MV. Chitin synthase inhibitors as antifungal agents. Mini Rev Med Chem 2013;13(2):222-36.

28. Verwer PE, ten Kate MT, Falcone FH, Morroll S, Verbrugh HA, Bakker-Woudenberg IA, et al. Evidence supporting a role for mammalian chitinases in efficacy of caspofungin against experimental aspergillosis in immunocompromised rats. PLoS One 2013;8(10):e75848.

29. Sanglard D, Coste A, Ferrari S. Antifungal drug resistance mechanisms in fungal pathogens from the perspective of transcriptional gene regulation. FEMS Yeast Res 2009;9(7):1029-50. 